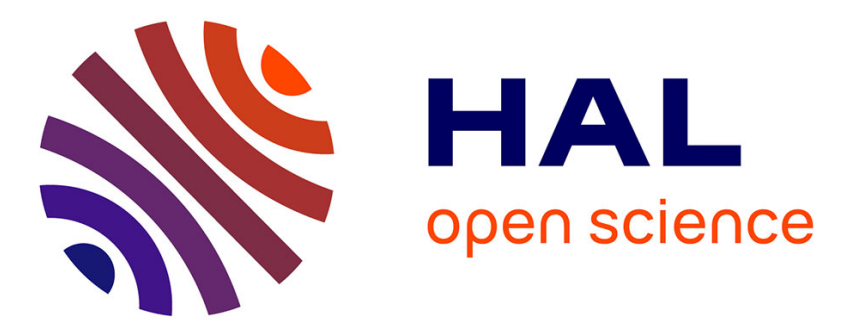

\title{
A survey on dynamic and stochastic vehicle routing problems
}

Ulrike Ritzinger, Jakob Puchinger, Richard F. Hartl

\section{To cite this version:}

Ulrike Ritzinger, Jakob Puchinger, Richard F. Hartl. A survey on dynamic and stochastic vehicle routing problems. International Journal of Production Research, 2016, 54 (1), 10.1080/00207543.2015.1043403 . hal-01224562

\section{HAL Id: hal-01224562 \\ https://inria.hal.science/hal-01224562}

Submitted on 6 Nov 2015

HAL is a multi-disciplinary open access archive for the deposit and dissemination of scientific research documents, whether they are published or not. The documents may come from teaching and research institutions in France or abroad, or from public or private research centers.
L'archive ouverte pluridisciplinaire HAL, est destinée au dépôt et à la diffusion de documents scientifiques de niveau recherche, publiés ou non, émanant des établissements d'enseignement et de recherche français ou étrangers, des laboratoires publics ou privés.

\section{(1)(1) $\$(0)$}

Distributed under a Creative Commons Attribution - NonCommercial - ShareAlikel 4.0 
To appear in the International Journal of Production Research

Vol. 00, No. 00, 00 Month 20XX, 1-19

\title{
A Survey on Dynamic and Stochastic Vehicle Routing Problems
}

\author{
Ulrike Ritzinger $^{\mathrm{a} *}$ and Jakob Puchinger ${ }^{\mathrm{a}}$ and Richard F. Hartl ${ }^{\mathrm{b}}$ \\ ${ }^{a}$ Mobility Department, AIT Austrian Institute of Technology, Austria; \\ ${ }^{b}$ Department of Business Administration, University of Vienna, Austria
}

(Received 00 Month 20XX; final version received 00 Month 20XX)

\begin{abstract}
Research on dynamic and stochastic vehicle routing problems received increasing interest in the last decade. It considers a novel problem class, aiming at an appropriate handling of dynamic events combined with the incorporation of stochastic information about possible future events. This survey summarizes recent literature in this area. Besides the classification according to the available stochastic information, a new classification based on the point in time where substantial computational effort for determining decisions or decision policies arises, is introduced. Furthermore, the difference in solution quality is analyzed between approaches which consider either purely dynamic or stochastic problems compared to those which consider both, stochastic and dynamic aspects. A graphical representation demonstrates the strength of the reviewed approaches incorporating dynamic and stochastic information. The survey also gives an overview on the intensity of research for the different problem classes and its benefit in recent years. Finally, guidelines and promising directions for further research are presented.
\end{abstract}

Keywords: Survey, Vehicle Routing Problem, Dynamic and Stochastic Aspects

\section{Introduction}

In the last years, research interest in vehicle routing has increasingly focused on dynamic and stochastic approaches. The advance of information and communication technologies as well as the growing amount of available data allow to gather relevant information for advanced vehicle routing. A crucial factor for being successful in solving sophisticated vehicle routing problems (VRP) is to offer reliable and flexible solutions. The recent development in telematics, such as the wide spread use of positioning services and mobile communication, allows gathering real-time information and exact monitoring of the vehicles. This builds the basis for extensive data collection and real-time decision support in vehicle routing. Additionally, today's computing capacity allows the application of routing algorithms which provide real-time solutions by incorporating online information and considering possible future events. These advances offer the opportunity to improve solution quality in real-time systems but they need to be leveraged by efficient optimization algorithms for solving dynamic and stochastic vehicle routing problems (DSVRP). Goel (2008), Larsen et al. (2008), Ehmke et al. (2012) demonstrate the development of technological environment and real-time management in vehicle routing.

This survey points out the relevance of using a combination of dynamic and stochastic information in the optimization of VRPs. The increasing interest in this area results in a fast growing body of research and multiple applications and settings arising from real-world problems. This leads to an unclear, sometimes confusing terminology and a lack of consistent problem formulations. Furthermore, there are almost no generalizable statements about the benefit of using dynamic and stochastic problem formulations compared to their pure counterparts. Besides a comprehensive

*Corresponding author. Email: ulrike.ritzinger@ait.ac.at 
overview of research on DSVRPs in recent years, the main contribution of this article is a classification and evaluation of the reviewed literature. The broad classification of this article relies on different stochastic parameters as in Gendreau et al. (1996). We introduce a novel characterization based on the optimization algorithms which distinguishes between methods relying on precomputed decisions and on methods performing online computation. Finally, the difference in solution quality is analyzed between approaches considering either purely dynamic or stochastic problems compared to those considering the combination of it.

This work starts with a summary of VRPs in Section 2, while the further sections examine dynamic VRPs with the focus on a particular stochastic aspect: travel times in Section 3, demand in Section 4, customers in Section 5, and problems with more than one stochastic parameter in Section 6. Finally, the survey is summarized and concluding remarks are given.

\section{Vehicle Routing Problems}

The VRP is an extensively studied but still challenging research topic. The categorization of VRPs based on the information availability and uncertainty is introduced in Schorpp (2010) and utilized in Pillac et al. (2013). This results in static, dynamic, stochastic, and dynamic and stochastic VRPs. Here, the focus is on dynamic and stochastic VRPs, but for the sake of completeness we give a short summary on dynamic VRPs and stochastic VRPs as well. For the static VRP, the interested reader is referred to an excellent overview of different VRP formulations in Toth and Vigo (2001) and other attractive reviews in Cordeau et al. (2007), Laporte (2007) and Laporte (2009).

\subsection{Dynamic Vehicle Routing Problems}

In many applications, not all information about the problem instance is known in advance. In the dynamic VRP (DVRP), also referred to as real-time or online VRP, some input data is revealed during the execution of the plan. The most common dynamic events in VRPs is the arrival of new customer requests but demands, service times and travel times are possible dynamic components as well. The DVRP is extensively studied in the literature, starting with the work of Psaraftis (1988) which demonstrates the differences between static and dynamic VRPs. Larsen and Madsen (2000) presents a classification of DVRPs according to the dynamism of the system, whereas Pillac et al. (2013) classify DVRPs regarding the type of dynamic events. Various algorithms handling DVRPs are proposed in the literature and excellent reviews on DVRPs are given in Psaraftis (1995), Larsen and Madsen (2000), Larsen et al. (2008), Jaillet and Wagner (2008), Schorpp (2010) and Pillac et al. (2013).

Involving dynamic information increases the complexity of the problem and new challenges emerge. The decision whether a new requests is accepted or rejected is introduced as service guarantee in Van Hentenryck and Bent (2009) and discussed in Ichoua et al. (2000), Li et al. (2009). Karsten Lund and Rygaard (1996) defines the degree of dynamism (DOD) which determines how dynamic a system is according to the number of dynamic request and the number total requests. Larsen and Madsen (2000) introduced further measurements which additionally consider the time aspect. Another challenge is to set up an appropriate objective function, since attributes such as service level, number of serviced requests, minimization of response times, or revenue maximization are concerned in contrast to simply considering travel times or distances. Since DVRPs require online decisions, a compromise between reactiveness and decision quality must be found. A highly accepted performance measure for online algorithms is the competitive analysis introduced by Sleator and Tarjan (1985) and further research on this topic is presented in Krumke (2002), Angelelli et al. (2007), Jaillet and Wagner (2008). The adjustment of the planned solution according to the plan in execution is another crucial factor and can be performed at certain points in time (event-based or time-driven) with different updating strategies. 


\subsection{Stochastic Vehicle Routing Problems}

In almost all real-world applications, uncertainty is an inherent characteristic of the problem. Usually, information about upcoming events is available through historical data, which can be converted into information models. The processes of data collection, analysis and provision is extensively discussed in Ehmke et al. (2012). The uncertainty in problem descriptions can be captured in various ways, mainly distinguishing between different formalization variants, as discussed in Bianchi et al. (2009). A general classification of stochastic combinatorial optimization problems (SCOP) is given according to the moment in time where uncertain information is revealed. This results in static SCOPs, where decisions are made before the random variables are realized and dynamic SCOPs, where decisions are taken after some random events have happened. The stochastic VRP (SVRP) is basically any VRP where one or more parameters are stochastic, meaning that some future events are random variables with a known probability distribution. A classification according to the stochastic parameters is proposed in Gendreau et al. (1996) and various optimization problem with uncertainty are summarized in Sahinidis (2004). Generally, pure SVRPs have a probability distribution of the random variables available and the optimization process is performed before they are realized. The planned routes are not changed or updated after the realization, thus, it is often referred to as a-priori optimization. One of the most commonly applied approaches to SVRPs is stochastic programming (SP) where a general introduction is given in Birge and Louveaux (1997) and SP in the context of transportation and logistics is discussed in Powell and Topaloglu (2003), Powell and Topaloglu (2005).

\subsection{Dynamic and Stochastic Vehicle Routing Problems}

The category of dynamic and stochastic VRPs (DSVRP) has given rise to increasing research interest in the last years. Due to recent advances in information and communication technologies, this novel problem class allows to handle real-world applications in a more accurate manner. The advantage is, that in addition to efficiently handling dynamic events, stochastic knowledge about the revealed data is considered. Flatberg et al. (2005) and Pillac et al. (2013) provide an overview on DSVRPs but with a strong focus on pure DVRPs, whereas Ritzinger and Puchinger (2013) give a review of DSVRPs but with an exclusive focus on various hybrid methods applied to this field. In recent years, the field of anticipatory optimization, dealing with the future realization of relevant parameters, has also been connected to dynamic decision making. A summary of successful methodologies for anticipatory optimization and dynamic decision making, categorized regarding different degrees of anticipation, is given in Meisel (2011).

The main contribution of this work, is a comprehensive overview and detailed classification on research considering DSVRPs. As already stated, the broad classification is based on the nature of the uncertain information as defined in Gendreau et al. (1996). However, the focus is on a further characterization based on the point in time where substantial computational effort for determining decisions or decision policies actually occurs. This results in two groups:

- The first group, preprocessed decisions, consists of approaches where policies or solutions are computed before the execution of the plan.

- The second group, online decisions, consists of approaches where solutions are computed as soon as a dynamic event occurs.

Both groups consider dynamic systems and are based on some stochastic information. In order to tackle such systems properly, state dependent decisions must be made, which is often named policy in the literature. The problem settings for both groups are usually modeled as Markov Decision Process (MDP) or formulated as multi-stage stochastic models. In Figure 1 the difference between the groups is illustrated.

Solution approaches which belong to the first group (preprocessed decisions) determine the values for decision making, respectively policies, before the execution of the solution plan. Therefore, 


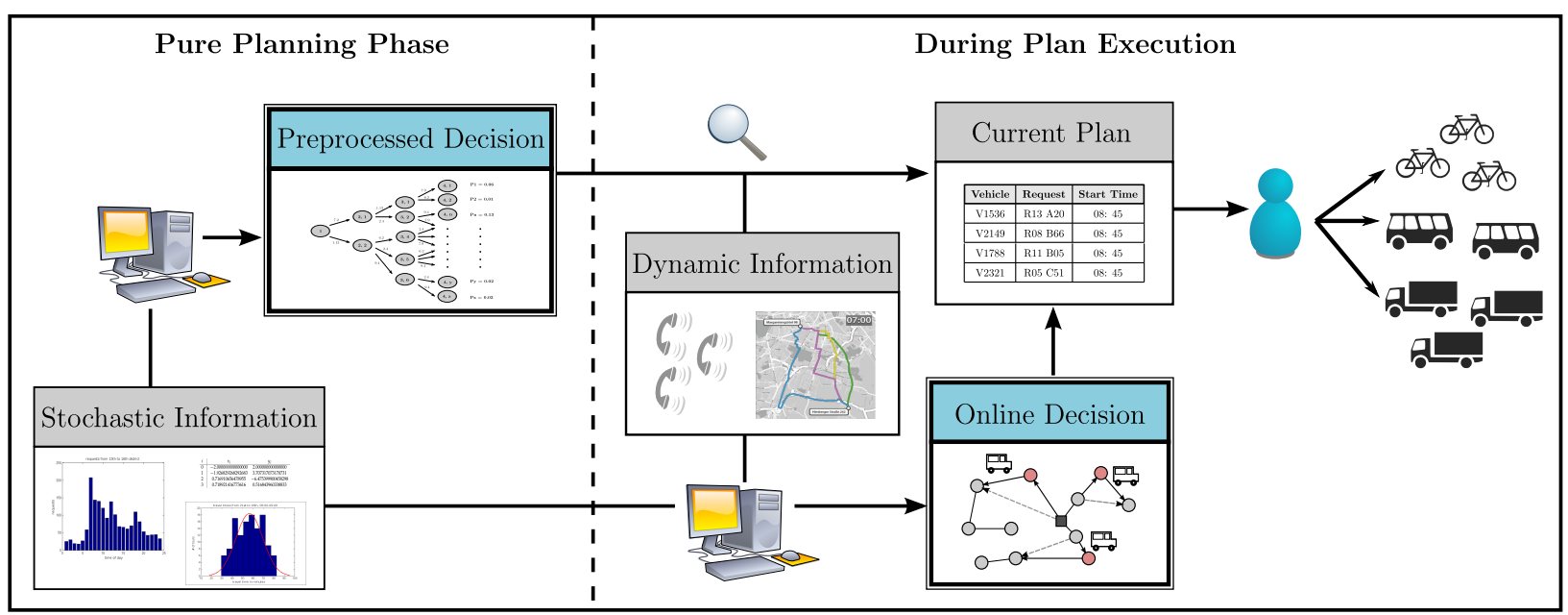

Figure 1. Illustration of the two groups: preprocessed and online decisions. The picture shows that preprocessed decision approaches spend most of the computational time processing stochastic information in the planning phase, before the actual execution of the plan. During the plan execution the current plan is updated based on look-ups to the preprocessed information. However, online decision approaches have to compute the results with respect to the dynamic and stochastic information during the operational process. Finally, a dispatching system receives an updated plan and is able to assign the vehicles.

possible states need to be constructed in advance and evaluated based on possible dynamic events and stochastic information along a considered time horizon. The first variant is to analyze general policies based on the arising states and decisions beforehand and apply them during the operational process, e.g. always dispatch the nearest available vehicle. The second variant, which is more relevant for this survey, is to value possible states and their decisions before plan execution and use these preprocessed values in the dynamic planning process. Thus, during plan execution these methods only exploit the precomputed values to make accurate decisions for the current system state. The second group (online decisions) differs in so far as a major part of the computation is made when a dynamic event occurs. Here, a current solution plan is followed during execution and whenever a new events arises a decision is calculated online with respect to the current system state and the available stochastic information. This procedure is also referred to as rolling horizon procedure or as look-ahead strategy. The solution of such an approach is either a single decision for the current situation or a re-optimized plan. Another possibility is to provide a greedy single decision requiring less computational effort first and run a more intensive re-optimization later in the background. The choice of the most adequate method to be used for reacting on dynamic events is strongly connected to the DOD and the given reaction time.

\section{DVRP with Stochastic Travel Times}

Travel times are fundamental data in VRPs, where the importance of accurate travel times for the considered network arises. Static deterministic travel times often exhibit very different characteristics in comparison with the real-world, since fluctuations in traffic density, most notably in urban areas, are not taken into account. In order to overcome this problem, there are the three possibilities to model travel times as time-dependent, stochastic, or stochastic and time-dependent. The latter variant is the combination of stochastic and time-dependent travel times and results in stochastic time-dependent networks, where link travel times are random variables with time-dependent distributions. Hall (1986) first studies the stochastic time-dependent shortest path problem, also called the least expected shortest path or least expected time path where a shortest path, based on estimation of mean and variance travel times, is constructed. The solutions are called a-priori or non-adaptive, since no decisions are updated once the vehicle is en route. Work on this can be found in Hall (1986), Fu and Rilett (1998) and Miller-Hooks and Mahmassani (2000). VRPs applying stochastic and time-dependent travel time are presented for example in $\mathrm{Fu}(2002)$, Chen 
et al. (2006), Woensel et al. (2008), Nahum and Hadas (2009), Lecluyse et al. (2009) and Taş et al. (2014). On the contrary, dynamic or adaptive solution approaches recognize the benefits of an adaptive decision making process and are investigated in this section.

\subsection{Preprocessed Decision Support}

In order to handle uncertainty and real-time information about travel times efficiently, travel times are analyzed and modeled appropriately and incorporated into the algorithms. Usually, this is modeled as a MDP and it is popular to model the stochastic information about travel times via probabilities of congestion on links. The aim is to calculate an optimal routing policy in advance which can then be applied to the vehicle en route. Note, that all the possible decisions are calculated and evaluated before the vehicle starts its route. One shortcoming is the dimension of the problems. So for every possible state regarding time, location and traffic information an optimal routing policy must be determined and stored. The computational effort can be demonstrated by the rather small test networks used in the literature. Interesting work which considers stochastic time-dependent travel times and which performs (optimal) routing decisions in advance is presented in $\mathrm{Fu}(2001)$, Miller-Hooks (2001), Kim et al. (2005), Gao and Chabini (2006), Gao and Huang (2012) and Güner et al. (2012). Recently, Toriello et al. (2014) propose an approximated linear programming (ALP) approach for the dynamic TSP with stochastic arc costs. The authors also investigate a rollout policy, where the expected costs at any state biased by the optimal value of the LP relaxation of a shortest Hamiltonian path are considered.

\subsection{Online Decision Making}

Here, the focus is on DVRPs with stochastic and time-dependent travel times where the decisions for adapting the route are not preprocessed but calculated online. In Taniguchi and Shimamoto (2004) and Potvin et al. (2006), the routes are updated according to estimated travel times whenever a vehicle arrives a customer location. In the former work, the travel times are obtained by a dynamic traffic simulation based on a macroscopic approach and results show the benefit of using the reactive algorithm compared to the static counterpart for test scenarios where a link is blocked for an hour at different times. In the latter case, travel times are obtained by a short-term forecast and dynamic perturbation model and a tolerance level, which is an allowed waiting time in case of lateness, before a reassignment action, is initiated. Results show that a good strategy is to accept lateness in case of small deviations in travel times, but react on events of a large magnitude. Yan et al. (2013) deal with the planning of courier routes and introduce a time-space network including several arcs associated with travel times and a probability. A stochastic planning model, incorporating unanticipated lateness penalty costs and a stochastic real-time adjustment model, which handles dynamic requests and aims for little route adjustment, are introduced. The results show, that the model with route adjustment yields better results. Another essential work is presented by Schilde et al. (2014), using stochastic deviations from time-dependent travel speeds which are deduced from historical accident data. The positive effect of using such data instead of average time-dependent travel speeds is tested on different stochastic metaheuristic concepts: dynamic stochastic variable neighborhood search (DSVNS) which is based on Gutjahr et al. (2007) and multiple scenario approach (MSA) introduced in Bent and Van Hentenryck (2004). The results are compared to the corresponding myopic approaches: a dynamic VNS (DVNS) and a multiple plan approach (MPA). In contrast to the work above, the true travel speed is revealed in each iteration of the algorithm. In general, the DSVNS performs best but the solution quality highly depends on the DOD in so far as for highly- and non-dynamic instances the DVNS works better. However, the MSA turns out to be unsuitable for this problem setting and, on average, obtains no improvements. 

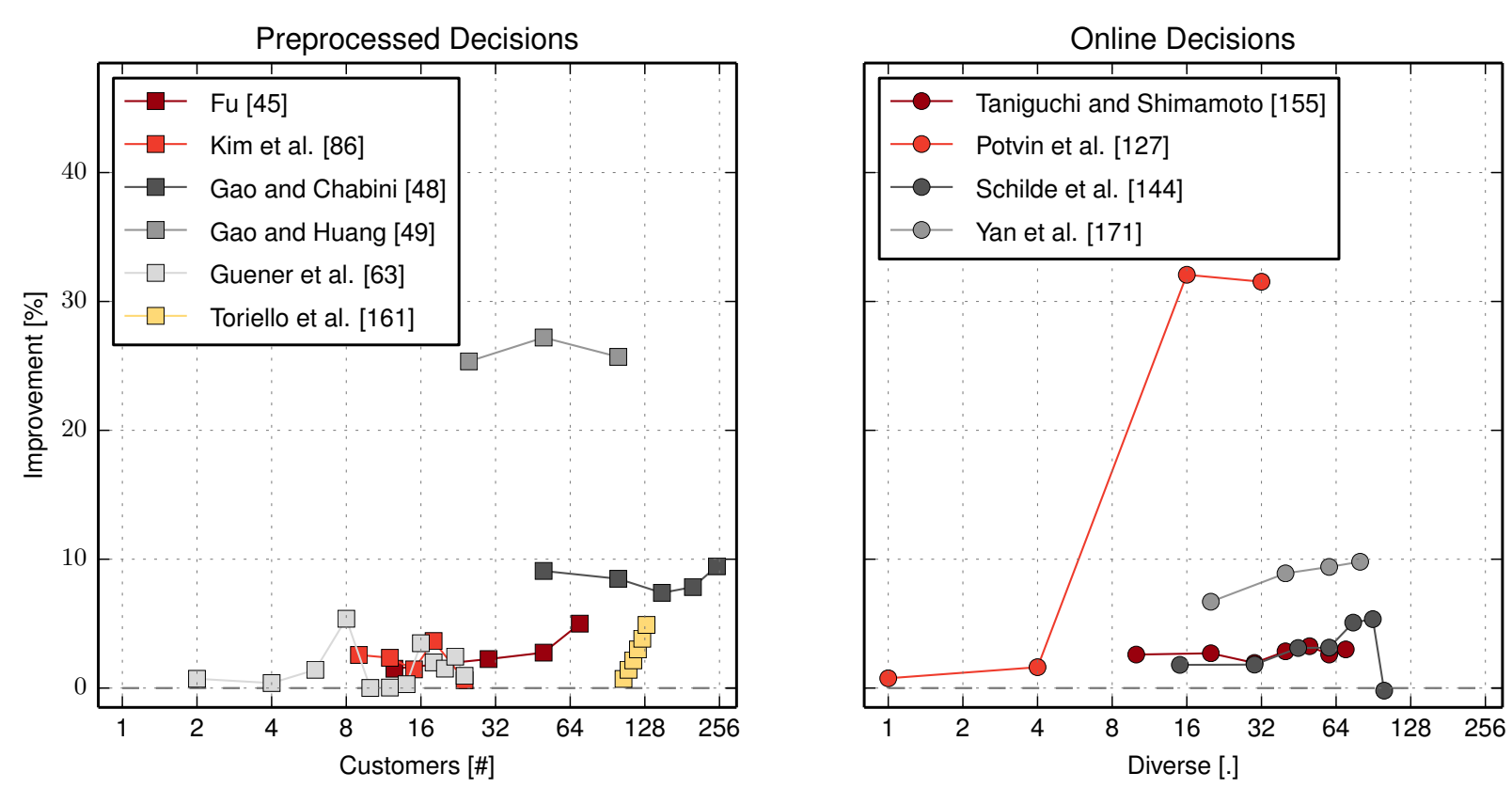

Figure 2. Illustration of the improvements of approaches for DVRPs with stochastic travel times compared to their static or myopic counterparts. Results for preprocessed decision algorithms (left) and online decision algorithms (right) clearly show the benefit with up to $10 \%$ improvement of incorporating uncertainty in travel times to the algorithms. Because no consistently presentable parameters are given for the instances of online algorithms, diverse measures (time, deviation, DOD) are shown.

\subsection{Conclusion}

Due to the problem specific constraints and the use of different test instances, the algorithms can not be fairly compared to each other. Nevertheless, each work demonstrates that even though the reduction of operational costs is not extensive, the reliability (i.e. level of customer service) increases considerably when uncertainty in travel times is considered in the solution approach. The stochastic and dynamic approaches are compared to static or myopic counterparts and the improvement regarding the main objective of the problem is illustrated in Figure 2. Note, that on the right side diverse measures are plotted because no consistently presentable parameter is given for the instances. Figure 2 clearly indicates that almost all improvements accumulate between $0 \%$ $10 \%$, letting us conclude this section with the statement that considering uncertainties of travel times in DVRPs allows the computation of more reliable routing plans. This increases customer satisfaction without a negative effect on operational costs.

\section{DVRP with Stochastic Demand}

Another intensively studied problem class is the VRP with Stochastic Demand (VRPSD), where uncertainty in the customers demand is given. The location of the customer is known in advance but the actual demand is revealed when arriving at the customer location. It occurs that the required demand can not be met and the vehicle has to return to the depot for replenishment before serving the customer. Therefore, the objective of VRPSD is to minimize the total expected travel costs needed to serve all customer demands. Typical applications for the VRPSD are the supply of gas stations or garbage collection. Usually, an a-priori solution which incorporates stochastic demand information is constructed and the customers are visited according to the plan without any route updates during the operation. Whenever the customer demand is not met a so-called route failure occurs and a defined recourse actions (e.g. replenishment at the depot) must be applied. Typically, a two-stage approach is applied as proposed in Bertsimas (1992) and Gendreau et al. (1996). The most common concepts are chance constraint programming (CCP) and stochastic programming with recourse (SPR). An excellent review on VRPSD is given in Campbell and Thomas (2008) and 
recent research on this topic is presented in Mendoza et al. (2010), Erera et al. (2010) and Juan et al. (2011), (2013). Anticipatory insertion (AI), discussed in Thomas and White III (2004), is another concept for exploiting future information about customer demand.

While a-priori solution approaches are intensively studied, advanced planning technology has also facilitated another approach where routing or replenishment decisions are made dynamically, often called re-optimization approach. This novel problem class is called DVRP with stochastic demand (DVRPSD). This section presents work on DVRPSDs which implies that VRPSDs are extended with the possibility of route adaption during the plan execution phase whenever new data is revealed. Additionally, the benefit of this novel combination is investigated.

\subsection{Preprocessed Decision Support}

A popular method for solving a DVRPSD is to consider all states (e.g. all possible demand realizations) in advance and value each state according to its performance. Such an approach performs the evaluation of the states before the vehicle starts the tour and enables an accurate decision making based on these values during the plan execution phase. From the point of computational effort it might be very expensive to determine all the predefined values, but it comes with the advantage that dynamic decisions can be provided quite fast. This is usually formulated as a multistage stochastic dynamic programming model and with the assumption that states and decisions are discrete the value evaluation can be done by Bellman's equation. To overcome the curse of dimensionality, the exact value function can be replaced by an approximation. This formulation can either be implemented as an ALP as in Toriello et al. (2014) or as an approximated dynamic programming (ADP) discussed in Powell (2007).

An ADP algorithm is discussed in Zhang et al. (2013) for the single-vehicle DVRPSD based on value function approximation (VFA). An ADP algorithm based on VFA with lookup table representation is developed and then improved by a Q-learning algorithm with bounded lookup tables and efficient maintenance. The algorithms are tested on the instances of Secomandi (2001) and Solomon (1987) and results show that especially for larger instances (up to 60 customers) the computational time could be reduced with the improved algorithm with even slight improvements in solution quality. Another ADP based approach is presented in Meisel et al. (2011) where the need of explicit anticipation of customer requests is discussed. The authors consider a single-vehicle approach, where the set of customers is divided into static and dynamic requests and for the latter either a rejection or acceptance decision is made after its arising. The algorithm is tested on the instances in Solomon (1987) and the results are compared the against the distribute waiting time strategy presented in Thomas (2007) and yield better results. To the best of our knowledge, these are the only two approaches for solving the DVRPSD reprocessing the values for dynamic decisions making in advance.

\subsection{Partly Online Decisions}

For the DVRPSD, this additional category is identified consisting of approaches where some of the computational effort is done in advance to guide the online decision making process. This evolves from the fact that all locations are known in advance and this information can be exploited for precalculations. A common sequential approach is the rollout algorithm (RA), which can be considered as a single iteration of policy iteration starting with a heuristically computed base policy. Based on its performance an improved policy is obtained by an one-step look-ahead. Thus, decisions for the current state are determined by approximating the cost-to-go via the base policy looking one-step ahead. Bertsekas (2013) provides an extensive survey on RAs and Goodson et al. (2012) describes rollout policies for general stochastic dynamic programs.

Secomandi $(2000,2001)$ provide the first computational results of a re-optimization policy for the DVRPSD by means of a RA. A one-step algorithm is developed where the cyclic-heuristic introduced in Bertsimas (1992) is used as base policy. Two versions (no-split and split delivery) are 
analyzed and compared to a static rollout approach. Secomandi and Margot (2009) develop an algorithm which determines re-optimization policies by computing the optimal policy for a restricted set of states (selected by a partitioning and sliding heuristic). Novoa and Storer (2009) extend the work in Secomandi (2001) by implementing different base policies. It is demonstrated that applying a two-step RA yields better results than the one-step RA. However, the best performing base policy is an adapted stochastic set-partitioning based model (Novoa et al. (2006)). Note, that these works consider the single-vehicle DVRPSD, whereas Fan et al. (2006) solve the multi-vehicle DVRPSD by decomposing it to single-vehicle problems first and applying the RA of Secomandi (2001) to each of them.

\subsection{Online Decisions}

Online decisions for the DVRPSD are determined either by applying online algorithms or, if computational time allows it, by recomputing the base sequence, at predefined states (e.g. an event arises). Early work is presented in Erera and Daganzo (2003) where the service region is divided into two parts and after serving all customers of the first part a single real-decision is made to assign the unserved customers to vehicles considering their remaining capacities. Pillac et al. (2012) propose an event-driven, sampling based MSA where a pool of scenarios is maintained. The scenarios are realizations of customer demands and are optimized by an adaptive VNS. The selection of the next customer is performed by a consensus function and results are compared to some large instances in Novoa and Storer (2009). Cheung et al. (2005) present a dynamic stochastic drayage problem where the duration of a task (transportation from origin to destination location combined with some intermediary activities) is considered to be uncertain. An adaptive labeling approach within a rolling horizon procedure is developed where virtual routes and labels are used and adapted to approximate the expected future costs. In Thomas (2007) intermediate requests at known locations may arise while the vehicle is en-route and waiting positions for serving these requests best possible are determined by a real-time heuristic, called center-of-gravity heuristic. A somehow surprising result is presented in Ghiani et al. (2012) where an anticipatory insertion heuristic (AI) is compared to a sample-scenario planning approach (MSA) for the dynamic stochastic TSP. The main contribution is to emphasize that anticipatory insertions, thus a-priori solutions, generate comparable solutions while needing less computational effort. Even tough the MSA shows poor performance, it has to be noted, that the MSA is sensitive to the problem structure (e.g. no time windows are given) and to instance data. As for Thomas (2007) it can be deduced, that an increase of the DOD has greater impact than an increase of the likelihood of requesting customers. In Goodson et al. (2013a), besides different a-priori based policies, a dynamic decomposition based rollout policy is presented to effectively tackle the multi-vehicle case. The customers are re-partitioned at each decision point by executing the fixed-route heuristic from the current state. In a further work, Goodson et al. (2013b) consider preemptive replenishment and present a RA in combination with a sampling based approach, where a sample average approximation is applied to estimate the expected value of a restocking policy along a fixed route to a set of scenarios. Recently, Zhu et al. (2014) introduce the paired cooperative re-optimization strategy formulated as a bilevel MDP, which makes use of partial re-optimization (Secomandi and Margot (2009)) and paired-vehicle cooperation (Ak and Erera (2007)).

\subsection{Conclusion}

Research shows the success of adaptive methods for the VRPSD (DVRPSD) compared to nonadaptive approaches. In Figure 3 the best obtained improvements of the algorithms are illustrated regarding the number of customers of the instances. Preprocessed decision approaches yield an average improvement of $0-5 \%$, whereas, RA based methods obtain up to $10 \%$ improvement. However, online decision approaches reveal the best benefit. Because different instances and recourse actions are applied, no fair comparison can be done among the algorithms but it has to be noted 

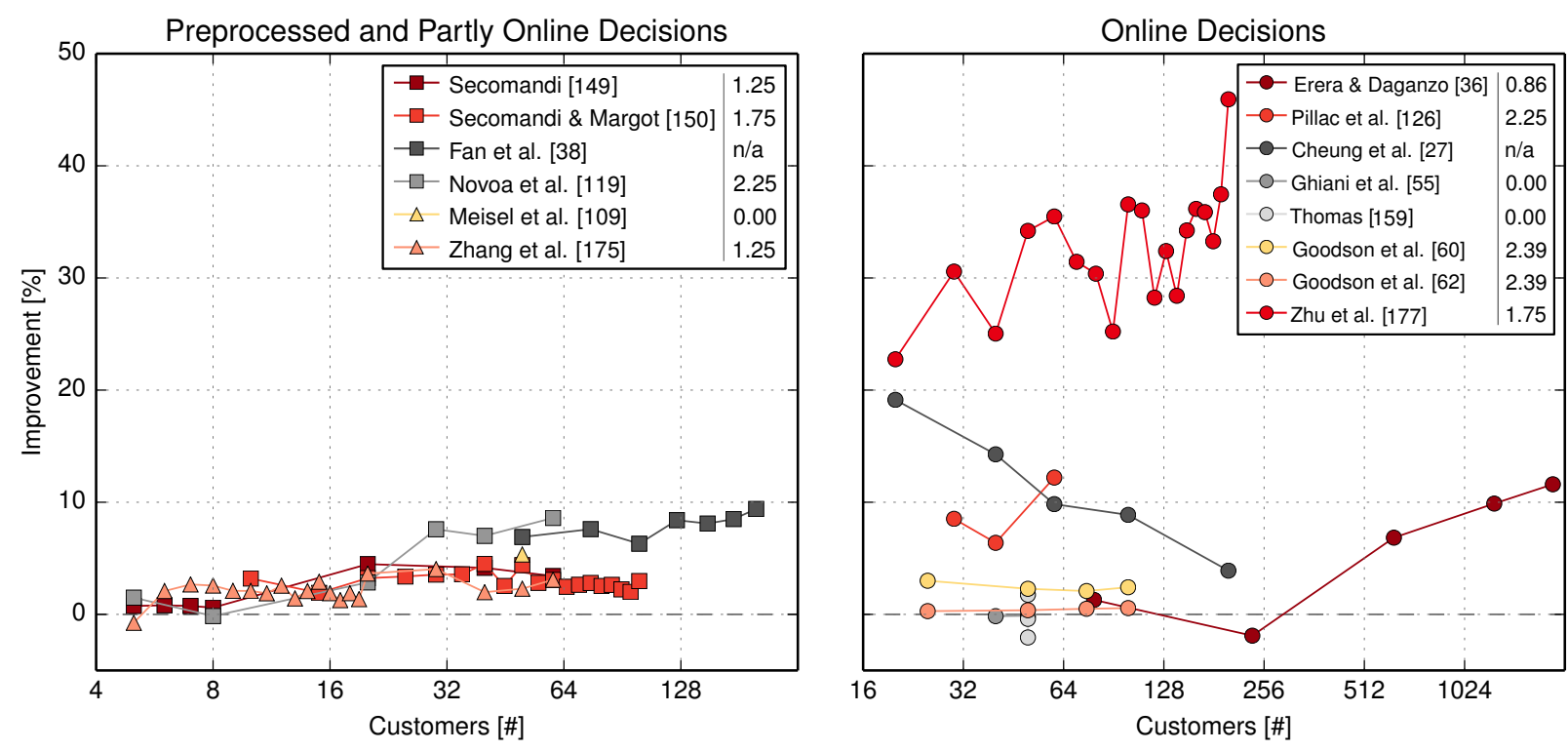

Figure 3. Illustration of the benefit on research of DVRPSDs compared to the static counterparts. On the left side results for preprocessed decision (marked as $\triangle$ ) and partly preprocessed decision (marked as $\square$ ) algorithms are shown and on the right side results for online decision (marked as o) algorithms are depicted. The possibility of re-optimization for the VRPSD yields decent improvements as shown by the results. Research on partly/preprocessed decision procedures is a little bit more explored but results on online decision methods show slightly better results.

that the instances in Novoa and Storer (2009) are publicly available and it is recommended to use them for comparison on future development. Nevertheless, the difficulty of problem instances which consider stochastic demand is measured by the expected filling rate (EFR), which is a ratio between the total expected demand and the total available vehicle capacity. According to Laporte et al. (2002) problem instances with an EFR $\geq 1$ can be considered as difficult. To demonstrate the complexity of the tested instances, the EFR is given in Figure 3 in the right column of the legend as well. EFR $=0$ means that a single-vehicle with no capacity restriction is given, whereas, in Fan et al. (2006) and Cheung et al. (2005) no description about the total available vehicle capacity is given. Concluding this section we state that, even though this problem class is rather new, a substantial number of papers on DVRPSD is available in the literature with a strong focus on single-vehicle problems. The trend is to investigate methods which are able to deal with more realistic problems, like larger sizes of instances as well as multi-vehicle VRPs.

\section{DVRP with Stochastic Customers}

Another well studied problem class is the DVRP with stochastic information about customers (DVRPSC). This problem class mainly arises in an environment, where some customer requests are known in advance but others are revealed during the day of operation. In contrast to the previous section, combined stochastic information about customer locations and the time of request occurrence is considered. This information is either available for each customer or it is aggregated geographically and/or temporally.

\subsection{Preprocessed Decision Support}

Besides myopic and look-ahead policies (also known as rolling horizon planning) and policy function approximation (PFA), a successful policy in operations research and the context of dynamic stochastic problems is value function approximation (VFA). In this context, ADP is a powerful framework for calculating the future impact of a decision and using an approximated value to improve it. This is demonstrated in an excellent tutorial on ADP in the field of transportation 
and logistic in Powell et al. (2012) and illustrated on different problem classes in Powell (2007). Godfrey and Powell $(2002,2002 b)$ present an ADP algorithm for a stochastic dynamic resource allocation problem with randomly arising tasks, whereas Spivey and Powell (2004) applied the ADP concept to dynamic assignment problems and Simão et al. (2009) present an ADP for dealing with a large-scale fleet management. Maxwell et al. (2010) and Schmid (2012) demonstrate the successful use of ADP algorithms for the dynamic ambulance relocation and dispatching problem under uncertainty. Other approaches using policies based on VFA are studied in Mes et al. (2013), Mes et al. (2010) where a decentralized auction based approach is considered and the exploit of incorporating historical job information and auction results into the planning process is investigated. Another research group studies the problem of designing motion strategies for mobile agents, where new customers arise randomly and remain active for a certain amount of time and the objective is to meet as many customers as possible within their active time window. The problem at hand is formulated as a DVRPSC and discussed in Pavone and Frazzoli (2010) and Pavone et al. (2009), Pavone et al. (2011).

\subsection{Online Decisions}

Based on the growing requirement to handle real-world problems efficiently, research efforts on approaches considering online decisions for the DVRPSC have increased in recent years. The aim is to appropriately react to new events by introducing rules that can be easily computed in realtime. These rules consider future events in the decision making process by generating scenarios of potential outcomes. A common concept, called sample scenario approach (SSA), is to generate multiple scenarios of future customer requests and include them into the planning process. After selecting the most appropriate solution for the future, the sampled customers are removed but with the effect that the new solution is well prepared for possible future requests.

Bent and Van Hentenryck (2004) introduce the MSA, an event-driven sampling based algorithm, where new solutions are generated regarding to a set of scenarios and maintained in the solution pool. The way of deciding which customer has to be visited next is essential for the MSA. Therefore, Van Hentenryck et al. (2010) present three algorithms sharing the same offline optimization algorithm and sampling procedure, but differing in the way of selecting the next customer at each decision step: the online expectation algorithm, the consensus algorithm and the regret algorithm. Further work is discussed in Bent and Van Hentenryck (2005), where approaches without distribution are presented: a machine learning approach to learn the distribution about requests during execution of the algorithm and a historical data approach, exploiting information about past instances and in Bent and Van Hentenryck (2007) where waiting and relocation strategies are investigated. Schilde et al. (2011) investigate the potential of using stochastic information about future return trips for the dynamic stochastic DARP applying two SSAs: a DSVNS and a MSA algorithm. It is shown that the incorporation of very near future information in the planning process (short-term sampling) is most beneficial. Another finding is that the DOD has a strong impact on the DSVNS, but the MSA is not affected as strongly. Another short-term SSA is presented in Ghiani et al. (2009), where near future requests are anticipated for a dynamic stochastic pickupand-delivery problem (PDP). Instead of using a scenario pool, an individual number of samples is determined and applied to the best alternative solutions. Out of them a distinguished solution is selected based on its expected penalty. Van Hemert and Poutré (2004) propose an evolutionary algorithm for the DVRPSC, where the samples consist of request occurring in fruitful regions, which are clusters of known customer locations that are likely to require service in near future. Flatberg et al. (2007) introduce an approach similar to the MSA but using a simple similarity score as consensus function. Additionally, the authors show how statistical knowledge of events can be learned automatically from the past, but no results are presented. Hvattum et al. (2006) formulate a multi-stage stochastic model and implement a dynamic stochastic hedging heuristic because of the problem size which is a time-driven SSA solving each sample scenario with a static algorithm 
and determining a plan based on common aspects of the generated solutions. Zhang et al. (2011) present a SSA and a capacity reservation approach, both designed as two-stage models within a rolling horizon framework for a multi-resource routing problem. In order to reduce computational effort a small set of decision epochs is defined and instead of using probabilistic models in the two-stage approach the sample average over a set of scenarios is optimized.

Besides sampling based approaches, other concepts like stochastic modeling or stochastic strategies (e.g. waiting strategies) are investigated for the DVRPSC. Yang et al. (2004) introduce a rolling horizon based real-time multi-vehicle truckload PDP and present a MIP formulation for the offline problem which is applied at every decision epoch in the online strategy. Another MIP based RH approach is presented in Kim et al. (2004) where dynamic decisions about the acceptance or rejection of requests are made based on an approximation of future vehicle utilization regarding spartial and temporal information about future requests. Larsen et al. (2004) present a rolling horizon approach where geographical and temporal aspects about customer requests are exploited in different strategies to reposition vehicles during idle times. In Ichoua et al. (2006), a PFA algorithm utilizes a threshold-based waiting strategy which decides how long a vehicle should wait at its last customer location according to the probability of future requests in this area. Sáez et al. (2008) present a hybrid adaptive predictive control approach based on a genetic algorithm where the demand pattern is obtained by a zoning method based on a fuzzy clustering model. Huth and Mattfeld (2011) present an algorithm which allows dynamic truck allocation for the stochastic swap container problem and investigate strategies for anticipating future demand realization. Results show that the use of a probability distribution is more beneficial than using expected values for anticipating future demands. Ferrucci et al. (2013) present a rolling horizon approach for the delivery of newspapers and apply a temporal and spartial clustering of future requests which guides vehicles into request-likely areas (cf. Van Hemert and Poutré (2004). It is claimed, that according to the comprised stochastic information of the clustering, solving only one scenario is adequate. Recently, Albareda-Sambola et al. (2014) present an adaptive service policy for a multi-period DVRPSC, where an auxiliary prize collecting VRP is solved at each time period. Additionally, a VNS based adaptive policy is applied to solve larger instances.

\subsection{Conclusion}

This section clearly shows that most research has been done on the DVRPSC for both categories. First, the potential of approaches based on VFA and the successful application of ADP algorithms for various problem structures is shown. Nevertheless, the main challenges are an accurate state formulation, the aggregation of data and the generation of scenarios to perform an extensive training phase. All of the presented work is tested on different real-world instances, which makes an objective comparison of the results difficult. However, the benefit of approaches belonging to this section, which is the improvements of considering stochastic information about customers compared to myopic algorithms, is depicted in Fig. 4 on the left side. The average improvement of the most successful method for every work is depicted in respect to the instance size (number of customers). Note, that the instance size in Simão et al. (2009) is 6000, but has been depicted at 600 for the sake of illustration.

Second, the research on online decision procedures distinguishes between non-sampling and sampling based approaches. Both methodologies demonstrate the benefit with up to $60 \%$ improvement of incorporating stochastic information about future customer requests into the solution algorithms as illustrated in Fig. 4 on the right side. Mainly, the algorithms are tested on adapted benchmark instances (Solomon 1987) or self-generated instances and in fact all papers focus on the comparison of their own myopic and anticipating approaches than comparing the results to other results in the literature. Note, almost all sampling based approaches are examined considering different levels of the DOD, except for Larsen et al. (2004) who present results for a non-sampling approach regarding the DOD. According to the advance of information and communication technologies future research 

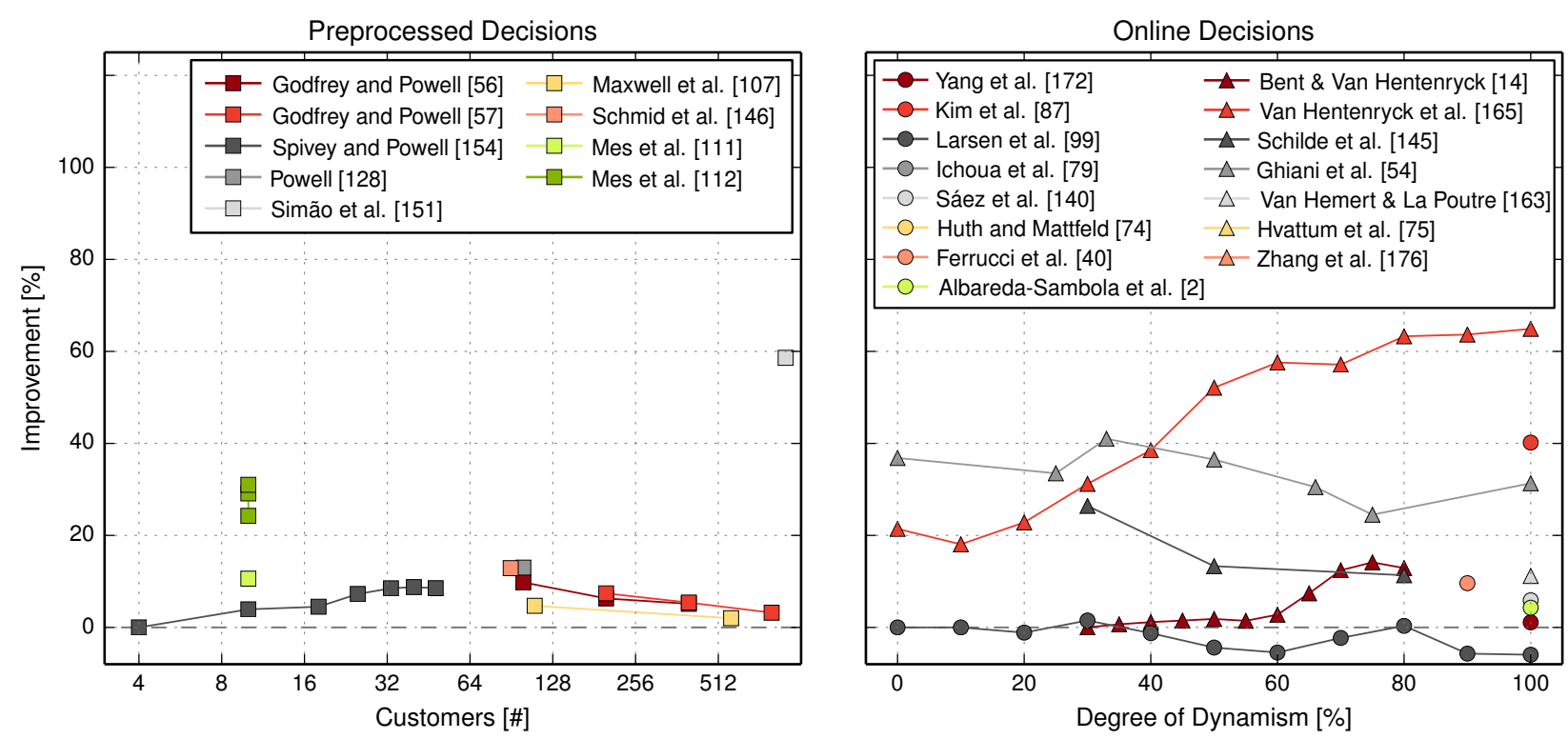

Figure 4. Illustration of the benefit of work on DVRPSC compared to their myopic counterpart. Sampling based approaches (marked as $\triangle$ ) are mostly examined on different levels of the DOD, non-sampling based approaches (marked as o) are not. The average results of the most successful approaches are depicted.

for both groups will be performed, however, it is assumed that sampling based approaches obtain more attention because of the advantage of not depending on an accurate probability distribution.

\section{DVRP with Multiple Stochastic Aspects}

Almost all research on DSVRPs considers not more than one stochastic aspect. Only little work is performed on investigating the impact of incorporating multiple stochastic aspects, e.g. consideration of stochastic travel times and customers. Based on the assumption that the availability of data will increase in the coming years and even better data analyses and probability models will become possible, knowledge about the impact and benefit of using more stochastic information in DSVRPs is essential. Moreover, research questions on the impact of various stochastic aspects, on the benefit of combinations of stochastic information, on the required level of detail have to be investigated. Furthermore, it has to be examined which settings work best for the various classes of DSVRPs. The small amount of research done so far on exploiting more than one stochastic element for DSVRPs is summarized in this section.

Cortés et al. (2008) extend the approach in Sáez et al. (2008) which considers stochastic customers, by additionally incorporating expected traffic conditions. The strategy approach is analyzed on scenarios with predictable and unpredictable congestion and results show an improvement of $2.1 \%$ compared to the myopic approach. In Bent and Van Hentenryck (2003) stochastic information about customers service times is considered and the MSA algorithm is applied. The aim is to investigate the behavior of the MSA algorithm on a less constrained but more stochastic problem. Results show that travel times are reduced while not degrading the service level and it is shown that the MSA is robust when stochastic information is not entirely accurate. Attanasio et al. (2007) present an approach with zoning technology for a same day courier service and introduce a forecast and an allocation module, where reliable near future predictions of travel times and demand are generated and handed to the allocation module which assigns customer requests and relocates idle couriers. Based on Hvattum et al. (2006), Hvattum et al. (2007) present a branch-and-regret heuristic (BRH) for the DVRPSC which finds better results regarding the number of vehicles used but not regarding the traveled distance. Furthermore, the performance is investigated when the demand of known customers is uncertain as well. The additional difficulty does not affect the performance of the myopic approach $(\mathrm{MDH})$, while the BRH performs worse when demands are 


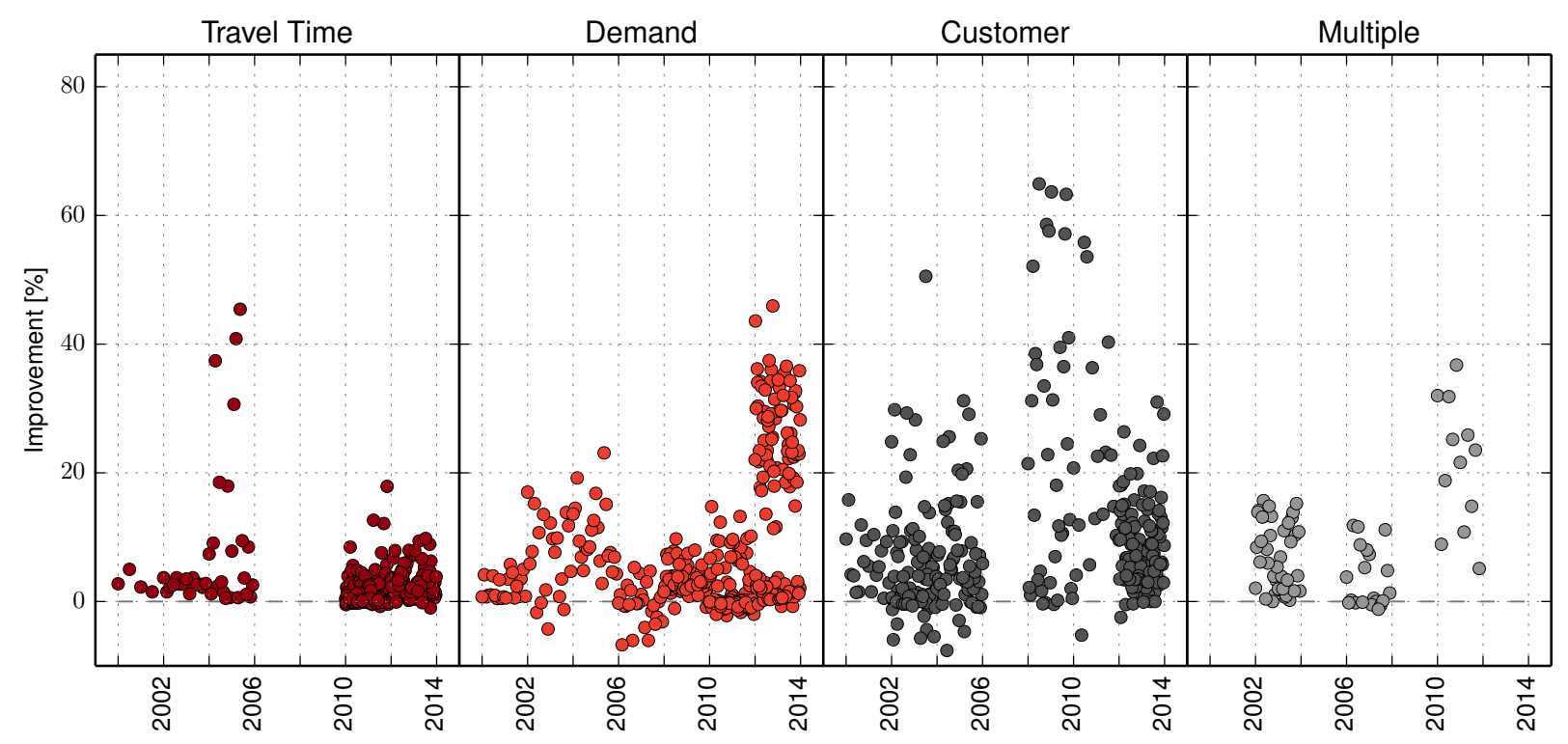

Figure 5. Illustration of all results on dynamic and stochastic VRPs grouped by the different stochastic aspects. Each group presents the results in the literature respecting the year of publication and its according improvement of the combination of dynamic and stochastic aspects compared to the myopic or deterministic counterparts.

stochastic, but performs better than the MDH but with a smaller gap. The algorithms are also compared to the best results in Bent and Van Hentenryck (2004), and it is shown that the MSA performs better than the BRH. Note, that the comparison is not completely fair, because the MSA is a event-based algorithm, which means that it is responded immediately to new requests, which is not the case in the time-driven BRH. Schilde (2012) did extensive research on the impact of using stochastic information during the planning process and demonstrates the effect of incorporating the combination of stochastic aspects (future transports and travel times) into the DSVNS and MSA algorithm. Results show that in contrast to the better performing DSVNS for approaches with a single stochastic aspect, the MSA outperforms the DSVNS when multiple stochastic aspects are considered. It is also shown that the combination leads to a more stable approach, indicating that increasing the amount of stochastic information has a positive effect on the robustness of the results.

Because in this section only results for the group of online decision procedures are reported, and due to space restrictions, a figure about results is omitted. However, to complete this section in Figure 5 the results of all discussed papers are summarized grouped according to the different stochastic aspects. The intensity of research for the different stochastic aspects is demonstrated and an overview of the overall performance of the categories is provided. Additionally, it gives an idea about the chronology of investigation on stochastic and dynamic routing problems. Figure 5 shows that research on DVRPs with stochastic travel times was mainly performed in the last years, whereas investigation on DVRPSD or DVRPSC is more equally distributed over the last decades and research on DVRPs with multiple stochastic aspects is rather scarce in the literature. Regarding the improvements achieved in the different classes, one can see that the benefit of considering dynamic and stochastic aspects in VRPs yields mainly up to 20\%, while the problem classes considering stochastic customer information perform very well with improvements up to 60 \%. Figure 5 clearly shows the benefit of considering dynamic and stochastic aspects in VRPs and should be an encouragement for research in this area in the future. 


\section{Conclusions}

Research interest in DSVRPs has increased considerably in the last years and recent technological development has facilitated real-time fleet management. First, it allows obtaining real-time information about the vehicles and their positions, traffic information, and up-to-date information about customer requests and respective demand. Second, novel telematics systems allow for the collection of large amounts of accurate data. The data can then be processed using modern statistics in order to provide useful stochastic information to the underlying optimization algorithms.

This survey has collected and classified relevant recent literature on DSVRPs. While existing review articles have either considered the class of DVRPs or the class of SVRPs, we focused on the combination of these two research fields and investigated its benefit. Moreover, a taxonomy of DSVRPs was presented, considering the types of available stochastic information, and their influence on the proposed solution approaches. In addition, a further characterization is identified respecting the point in time where substantial computational effort for the decision process is performed. Based on the reviewed research, one can conclude that the appropriate handling of dynamic events combined with the incorporation of stochastic information about possible future events typically yields better results compared to myopic or pure a-priori approaches. It should be noted, however that these approaches are computationally expensive which restricts, e.g. the number of scenarios that can be considered. On the other hand, already with today's state of the art personal computers, satisfactory results can be obtained within reasonable time. Clearly, technological progress will increase the potential of advanced methods even more.

Since telematics systems will further improve and become commonly used, the quality of the obtained data will increase, thereby allowing to provide more reliable information to optimization systems. For example, not only information from stochastic models using historical data, but also real-time information about traffic, requests, weather, etc. can be taken into account through machine learning techniques. This will allow treating more complex fleet management problems. On the one hand, problem size can increase and system interactions will become faster, and on the other hand, more complex and manifold data can be gathered, thus allowing for the combination of multiple stochastic aspects.

Further research could focus on gathering more reliable and robust information from the collected data and developing methods to combine several stochastic aspects. Here, the challenge will be to find out how much influence each individual parameter can have and how the interaction between these aspects will look like. Additionally, due to the increasing power and parallelization of computer processors the advantages of parallel algorithms could be exploited to deal with huge data and time-consuming methods, such as parameter estimation, sampling methods or background optimization procedures. In future, research questions considering the impact and limits of using complex stochastic information about multiple types of future events will increasingly gain importance.

Another direction of future research will emerge from the increasing demand for solving rich or multi-attribute VRPs. It can be expected that an increased amount of real-time and stochastic information will become available for such problems as well. Most of the developed methods for more classical dynamic and stochastic vehicle routing variants could be extended, but addressing complex constraints such as three-dimensional packing might require novel approaches.

While the relevance of stochastic and dynamic information for real world applications has been sufficiently documented, there is a certain research gap with respect to the comparability of results and approaches. More or less all surveyed papers deal with their own real world application or more precisely with their own artificial data based on real world applications. Most are not available to other researchers since the data are often "confidential". The papers typically compare one dynamic and stochastic approach (or sometimes a few variants implemented by themselves) with simple static and myopic re-optimization approaches and show that the more complex methods are somewhat better. But with very few exceptions it is hard to draw clear conclusions, which of the various alternative approaches surveyed here are the best. Hence, research should also focus 
on providing general, well documented, and publicly available test beds so that a more sound comparison is possible.

\section{Acknowledgements}

This work was partially funded by the Austrian Federal Ministry for Transport, Innovation and Technology (BMVIT) within the strategic program FIT-IT ModSim under grant 822739 (project HealthLog) and IV2Splus under grant 832423 (project SELECT).

\section{References}

Ak A, Erera AL (2007) A Paired Vehicle Recourse Strategy for the Vehicle Routing Problem with Stochastic Demands. Transportation Science 41(2):222-237

Albareda-Sambola M, Fernndez E, Laporte G (2014) The dynamic multiperiod vehicle routing problem with probabilistic information. Computers \& Operations Research 48(0):31-39

Angelelli E, Speranza MG, Savelsbergh MW (2007) Competitive analysis for dynamic multiperiod uncapacitated routing problems. Networks 49(4):308-317

Attanasio A, Bregman J, Ghiani G, Manni E (2007) Real-Time Fleet Management At Ecourier Ltd. In: Sharda R, Voß S, Zeimpekis V, Tarantilis CD, Giaglis GM, Minis I (eds) Dynamic Fleet Management, Operations Research/Computer Science Interfaces Series, vol 38, Springer US, pp 219-238

Bent R, Van Hentenryck P (2005) Online Stochastic Optimization without Distributions. In: Proceedings of the 15th International Conference on Automated Planning \& Scheduling, ICAPS 2005, AAAI Press

Bent R, Van Hentenryck P (2007) Waiting and Relocation Strategies in Online Stochastic Vehicle Routing. In: Proceedings of the 20th International Joint Conference on Artifical Intelligence, Morgan Kaufmann Publishers Inc., San Francisco, CA, USA, IJCAI'07, pp 1816-1821

Bent RW, Van Hentenryck P (2003) Dynamic Vehicle Routing with Stochastic Requests. International Joint Conference On Artificial Intelligence 18:1362-1363

Bent RW, Van Hentenryck P (2004) Scenario-Based Planning for Partially Dynamic Vehicle Routing with Stochastic Customers. Operations Research 52:977-987

Bertsekas DP (2013) Rollout Algorithms for Discrete Optimization: A Survey. In: Pardalos PM, Du DZ, Graham RL (eds) Handbook of Combinatorial Optimization, Springer New York, pp 2989-3013

Bertsimas DJ (1992) A Vehicle Routing Problem with Stochastic Demand. Operations Research 40(3):574585

Bianchi L, Dorigo M, Gambardella L, Gutjahr W (2009) A survey on metaheuristics for stochastic combinatorial optimization. Natural Computing 8:239-287

Birge JR, Louveaux F (1997) Introduction to Stochastic Programming. Springer New York

Campbell A, Thomas BW (2008) Challenges and Advances in A Priori Routing. In: Golden B, Raghavan S, Wasil E (eds) The Vehicle Routing Problem: Latest Advances and New Challenges, Operations Research/Computer Science Interfaces, vol 43, Springer US, pp 123-142

Chen HK, Hsueh CF, Chang MS (2006) The real-time time-dependent vehicle routing problem. Transportation Research Part E: Logistics and Transportation Review 42(5):383-408

Cheung RK, Hang DD, Shi N (2005) A labeling method for dynamic driver-task assignment with uncertain task durations. Operations Research Letters 33(4):411-420

Cordeau JF, Laporte G, Savelsbergh MW, Vigo D (2007) Chapter 6 Vehicle Routing. In: Barnhart C, Laporte G (eds) Transportation, Handbooks in Operations Research and Management Science, vol 14, Elsevier, pp 367-428

Cortés CE, Núñez A, Sáez D (2008) Hybrid adaptive predictive control for a dynamic pickup and delivery problem including traffic congestion. International Journal of Adaptive Control and Signal Processing 22(2):103-123

Ehmke JF, Steinert A, Mattfeld DC (2012) Advanced routing for city logistics service providers based on time-dependent travel times. Journal of Computational Science 3(4):193-205

Erera AL, Daganzo C (2003) A dynamic scheme for stochastic vehicle routing. Tech. rep., Georgia Institute of Technology, Atlanta, GA 
Erera AL, Morales JC, Savelsbergh M (2010) The Vehicle Routing Problem with Stochastic Demand and Duration Constraints. Transportation Science 44(4):474-492

Fan J, Wang X, Ning H (2006) A Multiple Vehicles Routing Problem Algorithm with Stochastic Demand. In: Intelligent Control and Automation, 2006. WCICA 2006. The Sixth World Congress on, vol 1, pp 1688-1692

Ferrucci F, Bock S, Gendreau M (2013) A pro-active real-time control approach for dynamic vehicle routing problems dealing with the delivery of urgent goods. European Journal of Operational Research 225(1):130141

Flatberg T, Hasle G, Kloster O, Nilssen EJ, Riise A (2005) Dynamic and Stochastic Aspects in Vehicle Routing - A Literature Survey. Technical Report STF90A05413, SINTEF ICT

Flatberg T, Hasle G, Kloster O, Nilssen EJ, Riise A (2007) Dynamic And Stochastic Vehicle Routing In Practice. In: Sharda R, Voß S, Zeimpekis V, Tarantilis CD, Giaglis GM, Minis I (eds) Dynamic Fleet Management, Operations Research/Computer Science Interfaces Series, vol 38, Springer US, pp 41-63

$\mathrm{Fu} \mathrm{L} \mathrm{(2001)} \mathrm{An} \mathrm{adaptive} \mathrm{routing} \mathrm{algorithm} \mathrm{for} \mathrm{in-vehicle} \mathrm{route} \mathrm{guidance} \mathrm{systems} \mathrm{with} \mathrm{real-time} \mathrm{information.}$ Transportation Research Part B: Methodological 35(8):749-765

Fu L (2002) Scheduling dial-a-ride paratransit under time-varying, stochastic congestion. Transportation Research Part B: Methodological 36(6):485-506

Fu L, Rilett LR (1998) Expected shortest paths in dynamic and stochastic traffic networks. Transportation Research Part B: Methodological 32(7):499-516

Gao S, Chabini I (2006) Optimal routing policy problems in stochastic time-dependent networks. Transportation Research Part B: Methodological 40(2):93-122

Gao S, Huang H (2012) Real-time traveler information for optimal adaptive routing in stochastic timedependent networks. Transportation Research Part C: Emerging Technologies 21(1):196-213

Gendreau M, Laporte G, Sguin R (1996) Stochastic vehicle routing. European Journal of Operational Research 88(1):3-12

Ghiani G, Manni E, Quaranta A, Triki C (2009) Anticipatory algorithms for same-day courier dispatching. Transportation Research Part E: Logistics and Transportation Review 45(1):96-106

Ghiani G, Manni E, Thomas BW (2012) A Comparison of Anticipatory Algorithms for the Dynamic and Stochastic Traveling Salesman Problem. Transportation Science 46(3):374-387

Godfrey GA, Powell WB (2002) An Adaptive Dynamic Programming Algorithm for Dynamic Fleet Management, I: Single Period Travel Times. Transportation Science 36(1):21-39

Goel A (2008) Fleet Telematics, Operations Research/Computer Science Interfaces Series, vol 40. Springer US

Goodson JC, Thomas BW, Ohlmann JW (2012) A generalized rollout policy framework for stochastic dynamic programming. Tech. rep., Saint Louis University

Goodson JC, Ohlmann JW, Thomas BW (2013a) Rollout Policies for Dynamic Solutions to the Multivehicle Routing Problem with Stochastic Demand and Duration Limits. Operations Research 61(1):138-154

Goodson JC, Thomas BW, Ohlmann JW (2013b) Restocking-Based Rollout Policies for the Vehicle Routing Problem with Stochastic Demand and Duration Limits. Tech. rep., University of Iowa

Güner AR, Murat A, Chinnam RB (2012) Dynamic routing under recurrent and non-recurrent congestion using real-time $\{$ ITS $\}$ information. Computers \& Operations Research 39(2):358-373

Gutjahr W, Katzensteiner S, Reiter P (2007) A VNS algorithm for noisy problems and its application to project portfolio analysis. In: Hromkovic J, Královic R, Nunkesser M, Widmayer P (eds) Stochastic Algorithms: Foundations and Applications, Lecture Notes in Computer Science, vol 4665, Springer Berlin/Heidelberg, pp 93-104

Hall RW (1986) The Fastest Path through a Network with Random Time-Dependent Travel Times. Transportation Science 20(3):182-188

Huth T, Mattfeld DC (2011) Myopic and Anticipated Planning in Stochastic Swap Container Management. International Journal of Operations Research 8(1):3-22

Hvattum LM, Løkketangen A, Laporte G (2006) Solving a Dynamic and Stochastic Vehicle Routing Problem with a Sample Scenario Hedging Heuristic. Transportation Science 40(4):421-438

Hvattum LM, Løkketangen A, Laporte G (2007) A Branch-and-Regret Heuristic for Stochastic and Dynamic Vehicle Routing Problems. Networks 49(4):330-340

Ichoua S, Gendreau M, Potvin JY (2000) Diversion Issues in Real-Time Vehicle Dispatching. Transportation Science 34(4):426-438

Ichoua S, Gendreau M, Potvin JY (2006) Exploiting Knowledge About Future Demands for Real-Time Vehicle Dispatching. Transportation Science 40(2):211-225 
Jaillet P, Wagner MR (2008) Online Vehicle Routing Problems: A Survey. In: Golden B, Raghavan S, Wasil E, Sharda R, Stefan V (eds) The Vehicle Routing Problem: Latest Advances and New Challenges, Operations Research/Computer Science Interfaces Series, vol 43, Springer US, pp 221-237

Juan A, Faulin J, Grasman S, Riera D, Marull J, Mendez C (2011) Using safety stocks and simulation to solve the vehicle routing problem with stochastic demands. Transportation Research Part C: Emerging Technologies 19(5):751 - 765

Juan A, Faulin J, Jorba J, Caceres J, Marquès J (2013) Using parallel \& distributed computing for real-time solving of vehicle routing problems with stochastic demands. Annals of Operations Research 207(1):43-65

Karsten Lund OBGM, Rygaard JM (1996) Vehicle Routing Problems with Varying Degrees of Dynamism. Technical report, IMM, The Department of Mathematical Modelling, Technical University of Denmark

Kim S, Lewis M, White I CC (2005) Optimal vehicle routing with real-time traffic information. Intelligent Transportation Systems, IEEE Transactions on 6(2):178-188

Kim Y, Mahmassani HS, Jaillet P (2004) Dynamic Truckload Routing, Scheduling, and Load Acceptance for Large Fleet Operation with Priority Demands. Transportation Research Record: Journal of the Transportation Research Board 1882:120-128

Krumke SO (2002) Online Optimization: Competitive Analysis and Beyond. Technical University of Berlin

Laporte G (2007) What you should know about the vehicle routing problem. Naval Research Logistics (NRL) $54(8): 811-819$

Laporte G (2009) Fifty Years of Vehicle Routing. Transportation Science 43:408-416

Laporte G, Louveaux FV, van Hamme L (2002) An Integer L-Shaped Algorithm for the Capacitated Vehicle Routing Problem with Stochastic Demands. Operations Research 50(3):415-423

Larsen A, Madsen OB (2000) The dynamic vehicle routing problem. Phd thesis, Technical University of Denmark

Larsen A, Madsen OB, Solomon MM (2004) The A Priori Dynamic Traveling Salesman Problem with Time Windows. Transportation Science 38(4):459-472

Larsen A, Madsen OB, Solomon MM (2008) Recent Developments in Dynamic Vehicle Routing Systems. In: Sharda R, Voß S, Golden B, Raghavan S, Wasil E (eds) The Vehicle Routing Problem: Latest Advances and New Challenges, Operations Research/Computer Science Interfaces Series, vol 43, Springer US, pp 199-218

Lecluyse C, Van Woensel T, Peremans H (2009) Vehicle routing with stochastic time-dependent travel times. 4OR: A Quarterly Journal of Operations Research 7:363-377

Li JQ, Mirchandani PB, Borenstein D (2009) A Lagrangian heuristic for the real-time vehicle rescheduling problem. Transportation Research Part E: Logistics and Transportation Review 45(3):419-433

Maxwell MS, Restrepo M, Henderson SG, Topaloglu H (2010) Approximate Dynamic Programming for Ambulance Redeployment. INFORMS Journal on Computing 22(2):266-281

Meisel S (2011) Anticipatory Optimization for Dynamic Decision Making, Operations Research/Computer Science Interfaces, vol 51. Springer, New York

Meisel S, Suppa U, Mattfeld D (2011) Serving Multiple Urban Areas with Stochastic Customer Requests. In: Kreowski HJ, Scholz-Reiter B, Thoben KD (eds) Dynamics in Logistics, Springer Verlag, pp 59-68

Mendoza JE, Castanier B, Guret C, Medaglia AL, Velasco N (2010) A memetic algorithm for the multicompartment vehicle routing problem with stochastic demands. Computers \& Operations Research $37(11): 1886-1898$

Mes M, van der Heijden M, Schuur P (2010) Look-ahead strategies for dynamic pickup and delivery problems. OR Spectrum 32:395-421

Mes M, van der Heijden M, Schuur P (2013) Interaction between intelligent agent strategies for real-time transportation planning. Central European Journal of Operations Research 21(2):337-358

Miller-Hooks E (2001) Adaptive least-expected time paths in stochastic, time-varying transportation and data networks. Networks 37(1):35-52

Miller-Hooks E, Mahmassani H (2000) Least expected time paths in stochastic, time-varying transportation networks. Transportation Science 34(2):198-215

Nahum O, Hadas Y (2009) Developing a model for the stochastic time-dependent vehicle-routing problem. In: Computers Industrial Engineering, 2009. CIE 2009. International Conference on, pp 118-123

Novoa C, Storer R (2009) An approximate dynamic programming approach for the vehicle routing problem with stochastic demands. European Journal of Operational Research 196(2):509-515

Novoa C, Berger R, Linderoth J, Storer R (2006) A Set-Partitioning-Based Model for the Stochastic Vehicle Routing Problem. Tech. Rep. 06T-008, Lehigh University

Pavone M, Frazzoli E (2010) Dynamic vehicle routing with stochastic time constraints. In: Robotics and 
Automation (ICRA), 2010 IEEE International Conference on, pp 1460-1467

Pavone M, Bisnik N, Frazzoli E, Isler V (2009) A stochastic and dynamic vehicle routing problem with time windows and customer impatience. Mobile Networks and Applications 14(3):350-364

Pavone M, Frazzoli E, Bullo F (2011) Adaptive and distributed algorithms for vehicle routing in a stochastic and dynamic environment. Automatic Control, IEEE Transactions on 56(6):1259-1274

Pillac V, Guéret C, Medaglia A (2012) An event-driven optimization framework for dynamic vehicle routing. Decision Support Systems 54(1):414-423

Pillac V, Gendreau M, Guéret C, Medaglia A (2013) A review of dynamic vehicle routing problems. European Journal of Operational Research 225(1):1-11

Potvin JY, Xu Y, Benyahia I (2006) Vehicle routing and scheduling with dynamic travel times. Computers \& Operations Research 33(4):1129-1137, part Special Issue: Optimization Days 2003.

Powell WB (2007) Approximate Dynamic Programming: Solving the Curses of Dimensionality (Wiley Series in Probability and Statistics). Wiley-Interscience

Powell WB, Topaloglu H (2003) Stochastic Programming in Transportation and Logistics. In: Ruszczynski A, Shapiro A (eds) Stochastic Programming, Handbooks in Operations Research and Management Science, vol 10, Elsevier, pp 555-635

Powell WB, Topaloglu H (2005) Fleet Management. Applications of Stochastic Programming 5:185

Powell WB, Simao HP, Bouzaiene-Ayari B (2012) Approximate dynamic programming in transportation and logistics: a unified framework. EURO Journal on Transportation and Logistics 1(3):237-284

Psaraftis HN (1988) Dynamic Vehicle Routing Problems. In: Golden B, Assad A (eds) Vehicle Routing: Methods and Studies, Elsevier Science Publishers B.V., pp 223-248

Psaraftis HN (1995) Dynamic vehicle routing: Status and prospects. Annals of Operations Research 61:143164

Ritzinger U, Puchinger J (2013) Hybrid Metaheuristics for Dynamic and Stochastic Vehicle Routing. In: Talbi EG (ed) Hybrid Metaheuristics, vol 434, Springer, pp 77-95

Sáez D, Cortés CE, Núñez A (2008) Hybrid adaptive predictive control for the multi-vehicle dynamic pick-up and delivery problem based on genetic algorithms and fuzzy clustering. Computers \& Operations Research $35: 3412-3438$

Sahinidis NV (2004) Optimization under uncertainty: state-of-the-art and opportunities. Computers \& Chemical Engineering 28(6-7):971-983

Schilde M (2012) Solution methods for the dynamic stochastic dial-a-ride problem with time-dependent travel speeds. Phd thesis, University of Vienna, Department of Business Administration

Schilde M, Doerner KF, Hartl RF (2011) Metaheuristics for the dynamic stochastic dial-a-ride problem with expected return transports. Computers \& Operations Research 38(12):1719-1730

Schilde M, Doerner K, Hartl R (2014) Integrating stochastic time-dependent travel speed in solution methods for the dynamic dial-a-ride problem. European Journal of Operational Research 238(1):18-30

Schmid V (2012) Solving the dynamic ambulance relocation and dispatching problem using approximate dynamic programming. European Journal of Operational Research 219(3):611-621

Schorpp S (2010) Dynamic Fleet Management for International Truck Transportation focusing on Occasional Transportation Tasks. Phd thesis, University of Augsburg, Faculty of Economics and Business Administration

Secomandi N (2000) Comparing neuro-dynamic programming algorithms for the vehicle routing problem with stochastic demands. Computers \& Operations Research 27(11-12):1201-1225

Secomandi N (2001) A Rollout Policy for the Vehicle Routing Problem with Stochastic Demands. Operations Research 49(5):796-802

Secomandi N, Margot F (2009) Reoptimization Approaches for the Vehicle-Routing Problem with Stochastic Demands. Operations Research 57(1):214-230

Simão HP, Day J, George AP, Gifford T, Nienow J, Powell WB (2009) An Approximate Dynamic Programming Algorithm for Large-Scale Fleet Management: A Case Application. Transportation Science 43(2):178-197

Sleator DD, Tarjan RE (1985) Amortized efficiency of list update and paging rules. Commun ACM 28(2):202208

Solomon MM (1987) Algorithms for the Vehicle Routing and Scheduling Problems with Time Window Constraints. Operations Research 35(2):254-265

Spivey MZ, Powell WB (2004) The Dynamic Assignment Problem. Transportation Science 38(4):399-419

Taniguchi E, Shimamoto H (2004) Intelligent transportation system based dynamic vehicle routing and scheduling with variable travel times. Transportation Research Part C: Emerging Technologies 12(3- 


\section{4):235-250}

Taş D, Dellaert N, Woensel TV, de Kok A (2014) The Time-Dependent Vehicle Routing Problem with Soft Time Windows and Stochastic Travel Times. Transportation Research Part C Accepted August 2014.

Thomas BW (2007) Waiting Strategies for Anticipating Service Requests from Known Customer Locations. Transportation Science 41(3):319-331

Thomas BW, White III CC (2004) Anticipatory Route Selection. Transportation Science 38(4):473-487

Toriello A, Haskell WB, Poremba M (2014) A dynamic traveling salesman problem with stochastic arc costs. Operations Research Forthcoming.

Toth P, Vigo D (eds) (2001) The Vehicle Routing Problem. Society for Industrial and Applied Mathematics, Philadelphia, PA, USA

Van Hemert J, Poutré J (2004) Dynamic Routing Problems with Fruitful Regions: Models and Evolutionary Computation. In: Yao X, Burke E, Lozano J, Smith J, Merelo-Guervós J, Bullinaria J, Rowe J, Tino P, Kabán A, Schwefel HP (eds) Parallel Problem Solving from Nature - PPSN VIII, vol 3242, Springer Berlin/Heidelberg, pp 692-701

Van Hentenryck P, Bent R (2009) Online Stochastic Combinatorial Optimization. The MIT Press

Van Hentenryck P, Bent R, Upfal E (2010) Online stochastic optimization under time constraints. Annals of Operations Research 177:151-183

Woensel TV, Kerbache L, Peremans H, Vandaele N (2008) Vehicle routing with dynamic travel times: A queueing approach. European Journal of Operational Research 186(3):990-1007

Yan S, Lin JR, Lai CW (2013) The planning and real-time adjustment of courier routing and scheduling under stochastic travel times and demands. Transportation Research Part E: Logistics and Transportation Review 53(0):34-48

Yang J, Jaillet P, Mahmassani H (2004) Real-Time Multivehicle Truckload Pickup and Delivery Problems. Transportation Science 38:135-148

Zhang C, Dellaert N, Zhao L, Woensel TV, Sever D (2013) Single Vehicle Routing with Stochastic Demands: Approximate Dynamic Programming. Tech. rep., Tsinghua University, Beijing, China

Zhang G, Smilowitz K, Erera A (2011) Dynamic planning for urban drayage operations. Transportation Research Part E: Logistics and Transportation Review 47(5):764-777

Zhu L, Rousseau LM, Rei W, Li B (2014) Paired cooperative reoptimization strategy for the vehicle routing problem with stochastic demands. Computers \& Operations Research 50(0):1-13 